\title{
Portfolio Selection Problem Considering Behavioral Stocks under Holding Periods
}

\author{
Kuo-Hwa Chang, Michael N. Young, and Wu Kang Lin
}

\begin{abstract}
The main objective of any investment is to earn but usually it's a long shot. In stock investments there are too much variables and unknowns that it's a challenge to make a sound decision. Any reliable information would be of great help to investors' portfolios. This study, introduces a possible solution for the information shortage by considering behavioral stocks (B-stocks). B-stocks are stocks that are positively affected by the collective irrational behaviors of investors. This led us to study the operational definitions (OD) of irrational behaviors and exploit the information such as the cause-effect patterns, time-to-effect, and the likelihood of the effect to occur. We consider the behavioral stock portfolio optimization problem (BSPO) wherein the causes of disposition effect and over-reaction B-stocks are spotted and are consequently bought and held according to the respective time-to-effect of each B-stock. With the likelihood of the effect to occur, the one-dimensional (SP/A) weighting function can be expanded into a two-dimensional (SP/A \& likelihood of effect) weightings function which is embedded to a scenario-based mixed integer program to solve the BSPO. The result shows that the portfolio can outperform the market significantly and that a possible new generic investment procedure can be exploited.
\end{abstract}

Index Terms-Behavioral stocks, portfolio optimization problem, operation research, weighting functions.

\section{INTRODUCTION}

Investors have different beliefs and opinions on how the market will play out in the future and how will they allocate their wealth. With their own views, they invest rationally according to sound technical analysis but more often than not they invest irrationally. Most investors tend to have their biases when allocating funds to investments. Some tend to follow the majority (herding behavior), some tend to over/under weight available market information (under/over-reaction), some see certain information (e.g. high book value, etc.) as signs (representativeness) of good investments, some tend to willingly invest their initial profits no matter the risk involve for a chance to earn more (house money effect), some tend to hold on to losers and realize winners quickly (disposition effect) and some have other tendencies like loss aversion or prospect theory, mental accounting, and so on. These tendencies have been heavily researched and are being studied by researchers which directly contradict the efficient market theory. Below are some of the studies about irrational behavior s and biases that

Manuscript received May 29, 2016; revised July 23, 2016. This work was partially supported by the Ministry of Science and Technology, Taiwan, ROC, under the grant contract MOST 103-2221-E-033 -023.

Kuo-Hwa Chang, Michael Nayat Young, Wu Kang Lin are with the Department of Industrial and Systems Engineering in Chung Yuan Christian University, Taiwan (e-mail: kuohwa@cycu.edu.tw, michaelnayatyoung@gmail.com,g10374037@cycu.edu.tw). are prevalent among investors.

Hot hand belief is the belief that a basketball shot made is more likely to go in if the previous shots also went in. [1] challenge this belief by studying professional basketball players and showed that the outcome of a shot is independent of the previous shots. [2] associated the hot hand belief in the tendency of investors to create trends even though in reality they do not exist. They discussed that wrong beliefs in basketball does not affect the next result, but wrong beliefs in stock markets, may influence the demand/supply for the stocks, which in turn affects the next asset prices.

Representativeness bias is the tendency of over-reliance to stereotypes or past events of similar environments. [3] explored a heuristic model for representativeness to which the subjective probability of an event, a sample, is determined by the degree of similarities in essential characteristics and features to its parent population. They demonstrated the representative heuristic show predictable and systematic errors in the evaluation of uncertain events. [4] asked specific people with representative bias which showed the disregard to prior probabilities when they were informed of similar events, but used the prior probabilities when they are not informed. [5] studied the relationship of the liked super bowl commercials to higher stock returns and found evidence of representative bias. [6] analyzed the Tunisian Stock Market and observed weak evidence of representative bias. [7] also analyzed the Tunisian Market and observed that representativeness bias is one of the many biases that influence the Tunisian investors in their decision making processes.

Over-confidence translates to too much belief in one's trading skill. [8] argued that over-confidence can explain high trading levels and the resulting poor performance among individual investors in U.S.A in the year 1991-1996. [9] observed that over-confident investors trade too much. They found out that among singles, men are more overconfident than women. This over-confidence exposes men to below average returns which are the result from excessive trading. In support to their initial findings that over-confident investors tend to be trading excessively, [10] analyzed the behavior of investors who shifted from phone trading to online trading and found out similar trend for over-confident investors. They reason that the shift to online trading allows investors to have wider access to information which makes the already over-confident investors to be more over-confident. [11] also showed evidences of over-confidence among investors. [12] investigates the link between the lack of consumer confidence and stock returns during market fluctuations and found out the lack of consumer confidence leads to higher probability of switching to a bear market regime. [13] found out that people believe 
that professional stock investors are more rational and over-confident than naïve investors.

Over / under - reaction is the tendency of individuals to over and under reaction to recent information. [14] showed evidence of over-reaction in a specific market with the findings that losers experience exceptionally large January returns after portfolio formation compared to winners. [15] further provided support for the existence of over-reaction as they studied the seasonal pattern of the returns which yielded similar findings that past losers significantly outperform past winners. [16] argued that over-reaction is as common as under-reaction, and that these anomalies are chance results.

Disposition Effect is the tendency of an investor to avoid loss and realize profit. [17] first coined the disposition effect wherein investors sell winners too early and hold losers too long. In support for the disposition effect, [18] provided evidence that overwhelmingly supports the disposition effect as determinant of year-end volume and volume levels throughout the year. [19] found out that professional investors and individual investors exhibit the disposition effect, although the effect is stronger for independent investors. [20] studied the prevalence of disposition effect at the Australian market and found out that it is more prevalent in investors of Chinese background, women, and older investors. They used frequent trading, round size trading heuristics, and the investor's level of diversification to predict the disposition effect.

These studies not only show that irrational behaviors exist among investors but also show that they affect the movement of the market and the underlying stocks within it. In this study, we determine how irrational behaviors affect individual stock returns, and then use the extra information to obtain an investor's optimal portfolio. The challenge will be similar to [21] on building superior portfolio based on the pricing distortions created by the collective emotional behavior of investors. Analyzing the available definitions of irrational behaviors, operational definitions (ODs) can be defined to detect which stocks are significantly affected by such biases. Detection can start by identifying the cause/trigger of the behavior, then determining the expected result or effect. The time before the effect will take place is also an important consideration as well as the likelihood of the effect to occur. The resulting cause - effect - time-to-effect likelihood-of-effect pattern will be very useful information for any investor which they can exploit for their portfolio optimization problem. Those stocks that exhibit the pattern are considered behavioral stocks that we call b-stocks. The likelihood-of-effect can also be considered in the weighting of future return scenarios to improve the available SP/A weighting functions and Prospect Theory-PT of [22] and [23], respectively, which will be embedded in the proposed optimization model. SP/A revolves around how fear and hope levels dictates the decision made by an individual, while PT revolves around the notion that investors value losses more compared to gains. Meaning in similar value of gain/loss, the individual will be much more troubled by the loss than he/she will be happy about the gains. PT was further improved by [24] into cumulative prospect theory where uncertainty is now treated cumulatively. [25] compared SP/A and CPT and claimed that SP/A is more suitable in modeling investment decision making in viewing the relation between descriptive and normative theories of risky choice. In this study, we consider SP/A and the likelihood-of-effect of the b-stock in our weighting function which we embed in our proposed optimization model.

In summary, this study will focus on the over-reaction and disposition effect b-stocks. The ODs for the said biases will be defined and the extra information will be exploited to obtain individual investors' optimal portfolios. Accordingly, we developed a portfolio optimization model that provides a procedure that will show how the extra information on b-stocks can be used to have a profitable portfolio. The resulting portfolio is compared to benchmarks like mean-variance model portfolio and the market portfolio.

The remainder of this paper is organized as follows. In Section II, discussions revolve on the ODs of over-reaction and disposition effect b-stocks, the investment pool considered and the portfolio model used to obtain the optimal portfolios. In Section III, description of the data used and how it is analyzed and interpreted the empirical results are presented. In Section IV, the conclusion, the contribution, and the possible future extension of the study are stated.

\section{Methodology}

In this study, we consider a daily investment in stock portfolios. We identify the daily investment pool composed of B-stocks from the initial pool of the 888 stocks in TWSE. Accordingly, we narrow it down twice to have our big and small pool of B-stocks. The procedure is discussed in the succeeding subsections.

\section{A. Operational Definition $(O D)$}

In this study we focus on over-reaction b-stocks and disposition effect b-stocks. The ODs are identified through the cause - effect - time-to-effect - likelihood-of-effect relationship of each stock. The cause is the trigger or the start of the behavior usually will depend on the bias, the favorable effect is to have a positive return (atleast $1 \%$ cumulative abnormal return), the time-to-effect is the time until the effect will likely occur, and the likelihood-of-effect is the probability that effect will take place after the time-to-effect.

Similar with [26] and following the description of [27] and [28] for over-reaction, the OD for over-reaction is define as "a large positive (negative) price movement followed by a high negative (positive) cumulative abnormal return, CAR. CAR is the summation of the abnormal returns, AR, for the desired number of time periods (time-to-effect) to be tested such that $\mathrm{CAR}=\sum_{i=1}^{t} A R_{t}$ where $A R_{t}$ is the abnormal return at time $\mathrm{t}$, such that $A R_{t}=R_{i t}$ $\left[\beta_{i t}\left(R_{M t}-R_{F t}\right)+R_{F t}\right]$, and since the daily risk-free rate, $\mathrm{R}_{\mathrm{Ft}}$ is close to 0 , for our preliminary test, we rewrite the abnormal return as $A R_{t}=R_{i t}-\beta_{i t} R_{M t}$, where $R_{i t}\left(R_{M t}\right)$ is the return rate of stock i (market) at time t, and $\beta_{i t}$ is the beta of stock $\mathrm{i}$ at time $\mathrm{t}$.

Following the description of [29] for disposition effect, the OD for the disposition effect starts with "the tendency of investors to sell winning stocks too soon and hold losing stocks too long". Accordingly, selling winning stocks too soon will cause high trading volume while holding the losing stocks too long will cause low trading volume. With reference to the works of [30] and [18] which used volume of 
stocks traded to detect the disposition effect, the relationship of the movements of price and relative volume to the market is analyzed.

We use the cumulative return rate and abnormal volume to view the price and volume movement respectively. The cumulative return rate is calculated as $R_{i}=\prod_{t=1}^{X}\left(R_{i t}+1\right)-$ 1 , where Rit is the return of stock $i$ at time $t$. While the abnormal volume is defined as $A V_{i t}=\widehat{V_{l t}}-\left(a+b V_{m, t}\right)$. The abnormal volume is derived from the relationship of the relative raw volume of stock and the relative raw volume of the market by regression model, $V=a+b V_{m}+\sigma$, where $\widehat{V_{l t}}$ $\left(V_{m, t}\right)$ is the observed volume of stock $i$ (market) at time $t$. Relative raw volume for stock $i$ at time $t$ is computed as $V_{i t}=\frac{\text { volume at time } t}{\text { moving average of the volume up to time } t}$ and relative raw volume for the market at time $t$ is computed as $V_{m, t}=$ market volume at time $t$ $\overline{\text { moving average of the market volume up to time } t}$. Accordingly, the average abnormal volume for $X$ periods, $\overline{A V}$, and cumulative return rate, $R_{i}$, for each stock is analyzed. Those stocks with combination of high (low) $R_{i}$ and high (low) $\overline{A V}$ are identified as winning (losing) stocks which are experiencing the effect of the bias of disposition effect with the additional condition that the resulting $C A R$ will be negative (positive). The reason for this is that the winning (losing) stocks are sold (bought) at a little bit lower (higher) price than the average by an investor which in turn affects the price downward (upward) as more and more investor do the same, resulting to a negative (positive) CAR .

Accordingly, as we want to earn profit, we only consider those over-reaction and disposition effect affected stocks that experiences the effect of positive $C A R$. Based on historical data and the ODs for both over-reaction and disposition effect, for each stock, we look at the cause, effect, and time-to-effect (T) pattern. If the likelihood of this pattern to hold over time is significant we consider that specific stock as a B-stock. To check this, let $P_{i}^{B}$ denote the likelihood for stock $i$ to hold the pattern, one-proportion is used with a null (alternative) hypothesis of $H_{0}: P_{i}^{B} \leq p\left(H_{a}: P_{i}^{B}>p\right)$. Each stock are tested for time-to-effect, $T=1$ to $T=20$, if there are multiple $T$ found, the smallest $T$ value is selected.

\section{B. B-stocks Investment Pool}

In this study, after the initial pool of stock from the market, those B-stocks that have significant cause-effect-T pattern are all considered in the Big Pool. Considering category 2 of the BSPO problem which will be discussed on the next sub-section, for each stock in the Big Pool, we spot those stocks that show the cause for the under-reaction and disposition effect $b$-stocks which are high negative returns and losing stocks, respectively. After the cause is spotted we are confident with probability $P_{i}^{B}$ that the effect will take place $T$ days after. Consequently, this B-stock is then considered in the Small Pool.

\section{Optimization Model}

With the cause-effect- $T-P^{B}$ pattern, we can have 2 categories of behavioral stock portfolio selection problem (BSPO). First, we can consider the problem when we know the time epoch (e.g. tomorrow) that effect $\left(C A R_{i} \geq 1 \%\right.$ translating to positive returns) will take place, we can maximize our portfolio in such a way that we can exploit the information and buy those stocks today that will have the effect tomorrow. We call this BSPO problem Category 1 which is already presented by Chang et al. (2015). Second, when we know the time epoch that the cause is spotted, we can maximize our portfolio in such a way that it will exploit the information that we are confident with $P^{B}$ that after $T$ days the effect $\left(C A R_{i} \geq 1 \%\right.$ translating to positive returns) will take place. We call this BSPO problem Category 2.

In this study, considering the BSPO problem category 2, after spotting the cause for the over-reaction and disposition effect b-stocks, we know that the effect $\left(C A R_{i} \geq 1 \%\right)$ would happen after $T_{i}$ days (time-to-effect) for each b-stock $i$ with the corresponding likelihood (probability for the effect to occur) $P_{i}^{B}$. An investor with this knowledge would desire for a higher $C A R$ over a given $T$ because they want to have the highest mean abnormal return in a given day. Intuitively, investors in the end will want to maximize the expected return over $T$ days, thus instead of maximizing $\frac{C A R i}{T i}, \frac{C R i}{T i}$ is maximized. In reference with the concept of efficient frontier, b-stocks in the small pool are further screened out if it falls below the efficient line $\left(\alpha+\beta T_{i} ; \alpha=p=0.5 ; \beta=\right.$ $0.02)$ to ensure an efficient $P_{i}^{B}-T_{i}$ portfolio as shown in Figure 1. Furthermore for simplicity and the purpose of preliminary testing, the portfolio selection only allows holding the minimum allowable shares. With consideration of the likelihood-of-effect $\left(P_{i}^{B}\right)$, SP/A weighting function of return scenarios, and the generic safety-first portfolio selection model, we write our BSPO category 2 model as follows:

Parameters:

$C R_{i}=$ cumulative return of stock $i$

$T_{i}=$ holding days of stock $i$

$n_{d}=$ the number of available B-stocks on day $d$

$R_{p}=$ return rate of the portfolio

$R_{L}=$ tolerance level of loss

$\gamma=$ threshold level for $P\left(R_{p}<R_{L}\right)$

$p_{j}=$ the probability of scenario $j^{\text {th }}$ scenario

$P i^{B}=$ the likelihood that the effect will occur after $t$ days

$P_{i}=$ price of stock $i$

Budget $_{d}=$ budget of day $d$

$M=$ a very large number

Decision Variables:

$\tau_{i} \quad$ a binary indicator whether b-stock $i$ is considered in the small pool for day $d$. When $\tau_{i}=1$, no b-stock $i$ is being held on day $d$, otherwise $\tau_{i}=0$

$x_{i} \quad$ a binary indicator whether the b-stock is bought or not; if $x_{i}=\left\{\begin{array}{rr}0 & \text { no share bought } \\ 1 & 1 \text { unit of share bought }\end{array}\right.$ note that 1 unit of share is 1000 shares

$w_{j}$ a binary indicator whether the $R_{p}<R_{L}$ on scenario $j$. If $w_{j}=1$, the portfolio return fall below the $R_{L}$, otherwise $w_{j}=0$

Model:

$$
\max =\sum_{i=1}^{n_{d}}\left(\frac{C R i}{T i}\right) x_{i}
$$

Subjected to the following constraints

$$
R_{p}-R_{L} \leq M w_{j}, j=1,2, \ldots, m
$$




$$
\begin{gathered}
\sum_{j=1}^{m} p_{j} w_{j} \leq \gamma \\
x_{i} \leq M \tau_{i}, i=1,2, \ldots, k \\
x_{i} \leq 1+\frac{1}{M}\left(P i^{B}-\left(\alpha+\beta T_{i}\right)\right) ; \alpha=0.5, \beta=0.02 \\
x_{i} \geq \frac{1}{M}\left(P i^{B}-\left(\alpha+\beta T_{i}\right)\right) ; \alpha=0.5, \beta=0.02 \\
\sum_{i=1}^{n d} x_{i} P_{i} \leq \text { Budget }_{d}
\end{gathered}
$$

Eqn. (1) is the objective function that maximizes the total cumulative return over the time-to-effect period. Eqn. (2) is a safety first constraint that determines whether $R_{p}<R_{L}$ on return scenario $j$. Eqn. (3) is also a safety first constraint that ensures that $P\left(R_{p}-R_{L}\right) \leq \gamma$. Eqn. (4) ensures that the b-stock bought on day $d$ is within the small pool. Eqns. (5) and (6) ensures that the b-stock bought is $P_{i}^{B}-T_{i}$ efficient. And eqn. (7) ensures that the portfolio is within the available budget.

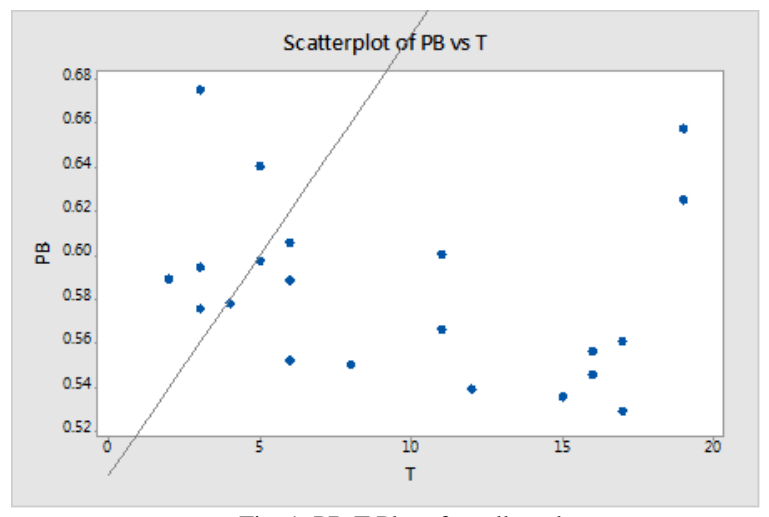

Fig. 1. PB-T Plot of small pool.

Only those stocks that are above the PB-T line are considered in the portfolio selection.

\section{EMPIRICAL RESULTS}

\section{Data Description}

From the Taiwan Economic Journal (TEJ), the closing prices and volumes of 888 stocks from the TWSE are obtained. These data are then used to calculate return rates and relative volumes for each stock, which are then screened to determine the big pool by 1 proportion test and the small pool by daily inspection. The collected data is from January 1, 1991 to December 31, 2015. The back-test period is from August 1, 2014 to December 31, 2015 for a total of 352 trading days. The category 2 BSPO model is then applied with the following parameters: cautiously hopeful investor fear and hope levels $(q s=q p=3, \gamma=0.5)$ with reference to Singer (2011), likelihood and time-to-effect $\left(P^{B}-T\right)$ linear inequality of $P i^{B} \leq 0.5+0.2 T_{i}$, initial capital of NT $\$ 5000000$, tolerance level $\left(R_{L}\right)$ of $-2 \%$, and a threshold level for the tolerance level $(\alpha)$ of $2 \%$. Overall, there are 3 portfolios compared to one another. All portfolios utilize the past 500 days historical data as their return scenarios. The portfolios are as follows:

BSPO denotes the portfolio using the BSPO category 2 model

MV denotes the portfolio using the mean-variance portfolio optimization model on the top 30 stocks of TWSE, with equal weights for mean and variance, and a daily budget of NT $\$ 5,000,000$

M denotes the corresponding daily returns of the TWSE market index

\section{E. Back-Test Results}

To evaluate the performances of the portfolios, with the assumption that the consideration of B-stocks would allow BSPO portfolio to outperform the benchmark (MV and M) portfolios, the portfolios are compared with one another. The portfolios' descriptive statistics, risk-adjusted returns, return distributions are first analyzed as shown in Table 1. The result shows that the BSPO portfolio can outperform the benchmark portfolios over the 352 days back-test period. BSPO portfolio dominates in all comparisons. BSPO portfolio has the highest daily mean return and has a volatility closest to the market as supported by the standard deviation and beta values. In terms of the risk-adjusted returns, BSPO portfolio still dominates the comparison with higher Sharpe and Treynor ratios. Looking at their return distribution the BSPO portfolio has the highest number of positive returns and the lowest number of negative returns. It also has the most instances of positive cumulative return with 192 out of the 352 days, while the MV and M portfolios have 160 and 170 days respectively. Intuitively, the BSPO portfolio also has the fewest instances of negative cumulative returns. It is evident from Table 1, that BSPO portfolio outperforms both MV and M Portfolio, and that no clear dominance can be seen comparing the MV and M portfolio.

TABLE I: DAILY DESCRIPTIVE RETURN STATISTICS, RISK-ADJUSTED RETURNS, AND RETURN DisTRIBUTIONS OF BSPO, MV, AND MARKET PORTFOLIOS

\begin{tabular}{llll}
\hline Descriptive Statistics & BSPO & MV & M \\
\hline Mean Return & 0.0010 & 0.0001 & -0.0003 \\
Standard Deviation & 0.0168 & 0.0205 & 0.0096 \\
Skewness & -0.2299 & 0.6891 & -0.3767 \\
Kurtosis & 2.3373 & 4.4965 & 2.7845 \\
\hline Risk-Adjusted Returns & BSPO & MV & M \\
\hline Beta & 0.9911 & 0.8655 & 1.0000 \\
Sharpe Ratio & 0.0601 & 0.0020 & -0.0388 \\
Treynor Ratio & 0.0010 & 0.0000 & -0.0004 \\
\hline Return Distribution & BSPO & MV & M \\
\hline Upside (R $>0)$ & 192 & 160 & 170 \\
Downside $(\mathrm{R}<0)$ & 160 & 191 & 182 \\
Breakeven $(\mathrm{R}=0)$ & 0 & 1 & 0 \\
Cumulative Return $(352$ Days) & 0.3756 & -0.0435 & -0.1243 \\
Cumulative Return $\geq 0$ & 252 & 78 & 121 \\
\hline
\end{tabular}

Another way to evaluate the performance of the portfolio is to check the daily accumulation of profit/loss. Fig. 2 shows the movement of the accumulated profit/loss over the 352 days of back-test. From the said figure, it is apparent that the movements of the accumulated profit/loss for the MV and M portfolios are very erratic while the movement of the accumulated profit/loss for the BSPO portfolio is consistent. As trading days increase there is accumulation of profit for the BSPO portfolio while the MV and M portfolios have irregular profit/loss.

To evaluate the performances of the portfolios in a more accurate way, Paired-T Tests are applied on the daily difference of each portfolio returns. Since the returns vary but depend on the same particular day, the analysis of daily 
difference on returns will eliminate the day effects. The alternative hypothesis for the test, $H_{a}$, is the average return pair difference is greater than 0 . The significant level to accept the alternative hypothesis is 0.1 . Note that if the $\mathrm{P}$-value is lower than 0.1 , it is confirmed that the average return pair difference is positive with $90 \%$ confidence. Conversely, if the P-value is higher than 0.1 , we fail to reject the null hypothesis, and that there is not enough evidence to support the alternative hypothesis. Accordingly, the results of the Paired-T Tests are shown in Table II.

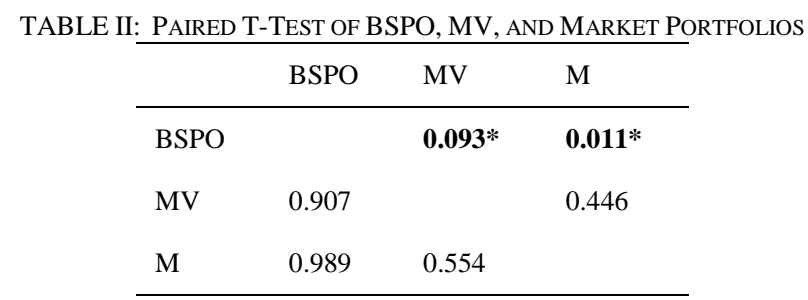

* denotes significant Paired-T-Test at $\alpha=0.1$

352 Days Cumulative Return (August 1, 2014 - December 31, 2015)

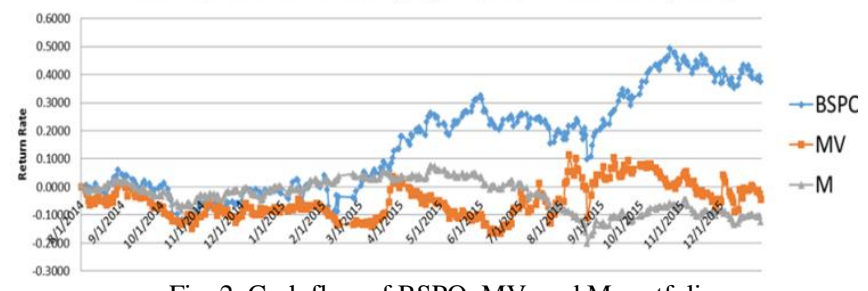

Fig. 2. Cash flow of BSPO, MV, and M portfolio.

Evidently, Table II shows with strong evidence that BSPO portfolio outperforms both MV and M Portfolios. While there is not enough evidence that MV portfolio outperforms BSPO and $\mathrm{M}$ portfolios, and there is also not enough evidence that $\mathrm{M}$ portfolio outperforms BSPO and MV portfolios.

\section{CONCLUSION}

The proposed behavioral stock portfolio optimization BSPO model utilizes the information of the cause/trigger, the positive effect, the time-to-effect, and the likelihood for the effect to occur. This study takes advantage of the information of knowing the time epoch when a cause/trigger is spotted and we are confident with the corresponding likelihood, $P^{B}$, that the stock would have the positive effect to its return after 1-20 days, time-to-effect. The proposed model ensures that the stocks bought satisfies the $P i^{B} \leq \alpha+\beta T_{i} ;(\alpha=$ $0.5 ; \beta=0.02$; linear inequality, which allows the portfolio to maximize the likelihood of the effect to occur at a given time-to-effect. From the back-test, the empirical result is consistent with the initial assumption that the extra information regarding b-stock would allow for a superior portfolio than the benchmarks. The BSPO portfolio shows dominance, stability, and profitability over the mean-variance and market portfolios. This study shows promising result, which can be duplicated by any investor and be considered a new generic way of investment.

This preliminary empirical study provides the following contributions and highlights: (1) the extra information regarding b-stocks is an advantage in investing; (2) the addition of the likelihood of the effect to occur in the weighting function improved the portfolio performance; (3) the flexibility of the model to cater other types of investors; (4) the profitability of the proposed model; and (5) the simplicity of the proposed model for easy duplication.

For future studies, extension can be made to improve the current model. Instead of just limiting the amount of stock that can be bought to the minimum allowable amount, the model can be expanded in such a way that it will allow for more shares but still within the available budget. Determining the best combination for the fear and hope levels to be used in the model is also a good extension, which if determined can be the generic parameters to be used in the proposed investment procedure. Lastly, the right frequency (daily, weekly, monthly, yearly transaction) of the transaction should also be determined to optimize the advantage of the B-stocks.

\section{ACKNOWLEDGMENT}

This work was partially supported by the Ministry of Science and Technology, Taiwan, ROC, under the grant contract MOST 103-2221-E-033 -023.

\section{REFERENCES}

[1] T. Gilovich, R. Vallone, and A. Tversky. (1985). The hot hand in basketball: On the misperception of random sequences. Cognitive Psychology. [Online]. 17(3). pp. 295-314. Available: doi:http://dx.doi.org/10.1016/0010-0285(85)90010-6

[2] M. Levy, H. Levy, and S. Solomon, 4 - Inefficient choices and investors' irrationality Microscopic Simulation of Financial Markets, San Diego: Academic Press, pp. 67-104, 2000.

[3] D. Kahneman and A. Tversky. (1972). Subjective probability: A judgment of representativeness. [Online]. Available: http://dx.doi.org/10.1016/0010-0285(72)90016-3

[4] A. Tversky and D. Kahneman, 2 - Judgment under Uncertainty: Heuristics and Biases: Biases in judgments reveal some heuristics of thinking under uncertainty, Academic Press, 1978.

[5] C. Chang, J. Jiang, and K. A. Kim, "A test of the representativeness bias effect on stock prices: A study of Super Bowl commercial likeability," Economics Letters, vol. 103, no. 1, pp. 49-51, 2009.

[6] R. Boussaidi, "Representativeness heuristic, investor sentiment and overreaction to accounting earnings: The case of the Tunisian stock market," Procedia - Social and Behavioral Sciences, vol. 81, pp. 9-21, 2013.

[7] Y. M. Rekik and Y. Boujelbene, "Determinants of individual investors' behaviors_evidence from Tunisian stock market," Journal of Business and Management, vol. 8, no. 2, pp. 109-119, 2013.

[8] B. M. Barber and T. Odean, "Trading is hazardous to your wealth: The common stock investment performance of individual investors," The Journal of Finance, vol. 55, no. 2, pp. 773-806, 2000.

[9] B. M. Barber and T. Odean, "Boys will be boys: Gender, overconfidence, and common stock investment," The Quarterly Journal of Economics, vol. 116, no. 1, pp. 261-292, 2001.

[10] B. M. Barber and T. Odean, "Online investors: Do the slow die first?" Review of Financial Studies, vol. 15, no. 2, pp. 455-488, 2002.

[11] R. J. Shiller, "Measuring bubble expectations and investor confidence," Journal of Psychology and Financial Markets, vol. 1, no.1, pp. 49-60, 2000.

[12] S. S. Chen, "Lack of consumer confidence and stock returns," Journal of Empirical Finance, vol. 18, no. 2, pp. 225-236, 2011.

[13] D. Peterson, A. Carlander, A. Gamble, T. Gärling, and M. Holmen, "Lay people beliefs in professional and naïve stock investors' proneness to judgmental biases," Journal of Behavioral and Experimental Finance, 2015.

[14] De Bondt, W. F. M., \& Thaler, R. (1985). Does the Stock Market Overreact? The Journal of Finance, 40(3), 793-805. doi:10.1111/j.1540-6261.1985.tb05004.x

[15] W. F. M. D. Bondt and R. H. Thaler, "Further evidence on investor overreaction and stock market seasonality," The Journal of Finance, vol. 42, no. 3, p. 557, 1987.

[16] E. F. Fama, "Market efficiency, long-term returns, and behavioral finance," Journal of Financial Economics, vol. 49, no. 3, pp. 283-306,1998. 
[17] H. Shefrin and M. Statman, "The disposition to sell winners too early and ride losers too long: Theory and evidence," The Journal of Finance, vol. 40, no. 3, pp. 777-790, 1985.

[18] S. P. Ferris, R. A. Haugen, and A. K. Makhija, "Predicting contemporary volume with historic volume at differential price levels: Evidence supporting the disposition effect," The Journal of Finance, vol. 43, no. 3, pp. 677-697, 1988.

[19] Z. Shapira and I. Venezia, "Patterns of behavior of professionally managed and independent investors," Journal of Banking and Finance, vol. 25 , no. 8, pp. 1573-1587, 2001.

[20] A. Frino, G. Lepone, and D. Wright, "Investor characteristics and the disposition effect," Pacific-Basin Finance Journal, vol. 31, pp. 1-12, 2015.

[21] C. T. Howard, Behavioral Portfolio Management: How Successful Investors Master their Emotions and Build Superior Portfolios, Harriman House Publishing, 2014.

[22] L. L. Lopes, "Between hope and fear: The psychology of risk: Wisconsin human information processing program," Department of Psychology, University of Wisconsin, 1987.

[23] D. Kahneman and A. Tversky, "Prospect theory: An analysis of decision under risk," Econometrica, vol. 47, no. 2, pp. 263-291, 1979.

[24] A. Tversky and D. Kahneman, "Advances in prospect theory: Cumulative representation of uncertainty," Journal of Risk and Uncertainty, vol. 5, no. 4, 1992.

[25] L. L. Lopes and G. C. Oden, "The role of aspiration level in risky choice: A comparison of cumulative prospect theory and SP/A theory," Journal of Mathematical Psychology, vol. 43, no. 2, pp. 286-313, 1999.

[26] K. H. Chang, M. N. Young, M. I. Hildawa, I. J. R. Santos, and C. H. Pan, "Portfolio selection problem considering behavioral stocks," in Proc. the World Congress on Engineering, 2015.

[27] D. R. Cox and D. R. Peterson, "Stock returns following large one-day declines: Evidence on short-term reversals and longer-term performance," The Journal of Finance, vol. 49, no. 1,1994.

[28] J. Madura and N. Richie, "Overreaction of exchange-traded fuds during the bubble of 1998-2002," Handbook of Behavioral Finance: Edward Elgar Publishing Limited, 2010.
[29] H. Shefrin and M. Statman, "The disposition to sell winners too early and ride losers too long: Theory and evidence," The Journal of Finance, vol. 40, no. 3, pp. 777-790, 1985

[30] E. A. Dyl, "Capital gains taxation and year-end stock market behavior," The Journal of Finance, vol. 32, no. 1, pp. 165-175, 1977.

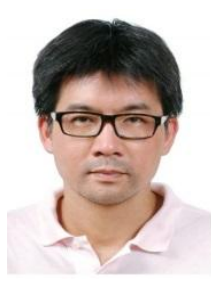

Kuo-Hwa Chang is a professor in the Department of Industrial and Systems Engineering in Chung Yuan Christian University, Taiwan. His current research interests include queueing system analysis on production systems and financial engineering.

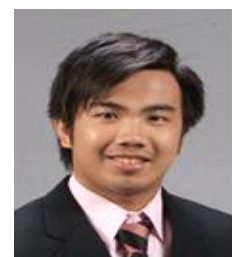

Michael N. Young is a Ph.D. student in the Department of Industrial and Systems Engineering in Chung Yuan Christian University, Taiwan. He is under the guidance of Dr. Chang and his research interests include financial engineering and queueing system analysis on production systems

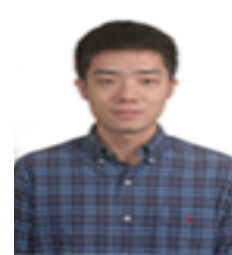

Wu Kang Lin is a master student in the Department of Industrial and Systems Engineering in Chung Yuan Christian University, Taiwan. He is under the guidance of Dr. Chang and doing his research interest on financial engineering. 